\title{
The Kirchhoff indices and the matching numbers of unicyclic graphs
}

\author{
Xuli Qi ${ }^{a}$, Bo Zhou ${ }^{b, *}$ Zhibin $\mathrm{Du}^{c}$ \\ ${ }^{a}$ Hebei Key Laboratory of Computational Mathematics \\ and Applications, and \\ College of Mathematics and Information Science, \\ Hebei Normal University, \\ Shijiazhuang 050024, P. R. China \\ ${ }^{b}$ Department of Mathematics, South China Normal University, \\ Guangzhou 510631, P. R. China \\ ${ }^{c}$ Department of Mathematics, Tongji University, \\ Shanghai 200092, P. R. China
}

\begin{abstract}
The Kirchhoff index of a connected graph is the sum of resistance distances between all unordered pairs of vertices in the graph. It found considerable applications in a variety of fields. In this paper, we determine the minimum Kirchhoff index among the unicyclic graphs with fixed number of vertices and matching number, and characterize the extremal graphs.
\end{abstract}

\section{Introduction}

The resistance distance was introduced by Klein and Randić [8] as a distance function on a graph. Let $G$ be a simple connected graph with vertex set $V(G)$ and edge set $E(G)$. The resistance distance between vertices $u$ and $v$ of $G$, denoted by $r_{G}(u, v)$, is defined as the effective resistance between nodes $u$ and $v$ of the electrical network for which nodes correspond to the vertices of $G$ and each edge of $G$ is replaced by a resistor of unit resistance (one ohm).

The Kirchhoff index of a connected graph $G$ is defined as [1]

$$
K f(G)=\sum_{\{u, v\} \subseteq V(G)} r_{G}(u, v) .
$$

\footnotetext{
${ }^{*}$ Corresponding author. Email: zhoubo@scnu.edu.cn
} 
It is also named as total effective resistance [6]. This graph invariant found applications in chemistry, electrical network, Markov chains, averaging networks, experiment design, and Euclidean distance embeddings, see [7, 1, 6].

The (ordinary) distance between vertices $u$ and $v$ of a graph $G$, denoted by $d_{G}(u, v)$, is the length of a shortest path connecting them in $G$. Recall that the Wiener index of $G$ is defined as [4, 5] $W(G)=\sum_{\{u, v\} \subseteq V(G)} d_{G}(u, v)$. It has been shown [8] that $r_{G}(u, v) \leq$ $d_{G}(u, v)$ with equality if and only if there is a unique path connecting $u$ and $v$ in $G$. As a consequence, the Kirchhoff index for a tree is equal to its Wiener index, which has been extensively studied (see [4]). Thus the Kirchhoff index is primarily of interest in the case of cycle-containing graphs.

Zhou and Trinajstić [13, 14] established various lower and upper bounds for the Kirchhoff index, see also [15]. Among the $n$-vertex connected graphs, Lukovits et al. [9] showed that the complete graph $K_{n}$ is the unique graph with minimum Kirchhoff index, and Palacios [10] showed that the path $P_{n}$ is the unique graph with maximum Kirchhoff index. The maximum and minimum Kirchhoff indices among the unicyclic graphs have been determined by Yang and Jiang [11], see also [12].

A matching $M$ of the graph $G$ is a subset of $E(G)$ such that no two edges in $M$ share a common vertex. A matching $M$ of $G$ is said to be maximum, if for any other matching $M^{\prime}$ of $G,\left|M^{\prime}\right| \leq|M|$. The matching number of $G$ is the number of edges of a maximum matching in $G$. For a matching $M$ of a graph $G$, if the vertex $v \in V(G)$ is incident with an edge of $M$, then $v$ is said to be $M$-saturated. Moreover, if every vertex of $G$ is $M$-saturated, then $M$ is a perfect matching of $G$.

Zhou and Trinajstić [16] determined the graphs with minimum Wiener index and Kirchhoff index respectively among the connected graphs with fixed number of vertices and matching number. Du and Zhou [3] determined the graphs with minimum Wiener index among the trees and unicyclic graphs respectively with fixed number of vertices and matching number.

In this paper, we determine the minimum Kirchhoff index among the unicyclic graphs with fixed number of vertices and matching number, and characterize the extremal graphs. It is of interest to point out that among the unicyclic graphs with fixed number of vertices and matching number, the graphs with minimum Kirchhoff index are different from those with minimum Wiener index (see [3]).

\section{Preliminaries and Lemmas}

For a graph $G$ with $v \in V(G), G-v$ denotes the graph resulting from $G$ by deleting $v$ (and its incident edges). For an edge $u v$ of the graph $G$ (the complement of $G$, respectively), $G-u v(G+u v$, respectively) denotes the graph resulting from $G$ by deleting (adding, respectively) $u v$. 
For $u \in V(G)$, let $K f_{G}(u)=\sum_{v \in V(G)} r_{G}(u, v)$. Then

$$
K f(G)=\frac{1}{2} \sum_{u \in V(G)} K f_{G}(u)
$$

Let $C_{n}$ be the cycle on $n \geq 3$ vertices, whose vertices are labeled consecutively by $v_{1}, v_{2}, \ldots, v_{n}$.

For two vertices $v_{i}, v_{j} \in V\left(C_{n}\right)$ with $i<j$, by Ohm's law, we have

$$
r_{C_{n}}\left(v_{i}, v_{j}\right)=\frac{(j-i) \cdot[n-(j-i)]}{n} .
$$

Furthermore, for fixed $n, r_{C_{n}}\left(v_{i}, v_{j}\right)$ is increasing for $j-i \leq\left\lfloor\frac{n}{2}\right\rfloor$. For $v_{1} \in V\left(C_{n}\right)$, by Eq. (1), we have

$$
K f_{C_{n}}\left(v_{1}\right)=\sum_{i=2}^{n} r_{C_{n}}\left(v_{1}, v_{i}\right)=\sum_{i=2}^{n} \frac{(i-1) \cdot[n-(i-1)]}{n}=\frac{n^{2}-1}{6}
$$

and thus

$$
K f\left(C_{n}\right)=\frac{1}{2} \cdot n \cdot K f_{C_{n}}\left(v_{1}\right)=\frac{n^{3}-n}{12} .
$$

For a unicyclic graph $G$ with the unique cycle $C_{k}, G-E\left(C_{k}\right)$ consists of $k$ vertexdisjoint trees $T_{1}, T_{2}, \ldots, T_{k}$, where $v_{i} \in V\left(T_{i}\right)$ for $i=1,2, \ldots, k$. These trees are called the branches of $G$, and $v_{i}$ is called the root of the branch $T_{i}$ in $G$ for $i=1,2, \ldots, k$.

Now we define the graph $U(k, t, i, j)$ which will be used frequently later. For integers $k, t, i, j$ with $k \geq 3, k \geq t \geq 0, i \geq 0, j \geq 0$, let $U(k, t, i, j)$ be the graph obtained from the cycle $C_{k}$ as follows:

(a) choose $t$ consecutive vertices in the cycle $C_{k}$;

(b) attach $t$ pendent vertices each to one of the $t$ chosen vertices in (a);

(c) attach $i$ pendent vertices and $j$ paths on two vertices to a central vertex of the $t$ chosen vertices in (a).

Clearly, $U(k, t, i, j)$ has $k+t+i+2 j$ vertices. In particular, let $U(k, t)=U(k, t, 0,0)$ for integers $k, t$ with $k \geq 3$ and $k \geq t \geq 0$. For example, $U(3,1,0,3), U(3,2,2,1)$ and $U(3,3,1,1)$ are shown in Fig. 1 .

Let $d_{G}(v)$ be the degree of $v$ in $G$.

For integers $n$ and $m$ with $2 \leq m \leq\left\lfloor\frac{n}{2}\right\rfloor$, let $\mathbb{U}(n, m)$ be the set of unicyclic graphs with $n$ vertices and matching number $m$. For integer $m \geq 2$, we can partition $\mathbb{U}(2 m, m) \backslash\left\{C_{2 m}\right\}$ into two subsets as follows: 


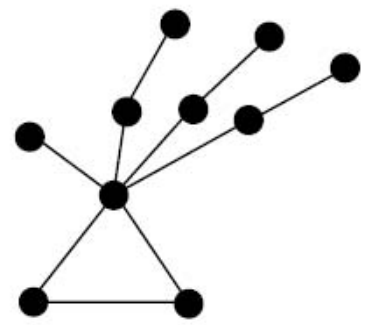

$U(3,1,0,3)$

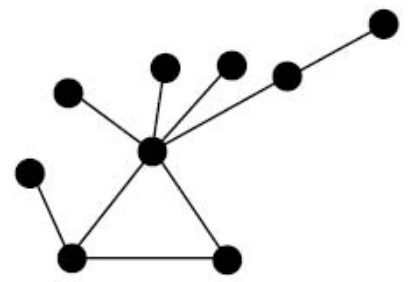

$U(3,2,2,1)$

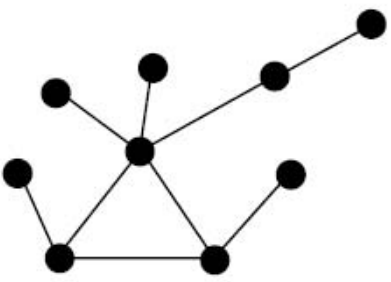

$U(3,3,1,1)$

Figure 1: The graphs $U(3,1,0,3), U(3,2,2,1)$ and $U(3,3,1,1)$.

(i) the set of graphs of maximum degree three in $\mathbb{U}(2 m, m)$ obtainable by attaching some pendent vertices to a cycle, which is denoted by $\mathbb{U}_{1}(m)$;

(ii) the set of graphs in $\mathbb{U}(2 m, m)$ containing some pendent vertex whose unique neighbor is of degree two, which is denoted by $\mathbb{U}_{2}(m)$.

\subsection{The Kirchhoff index of graphs in $\mathbb{U}_{1}(m)$ with small $m$}

First we want to determine the minimum Kirchhoff index among the graphs in $\mathbb{U}_{1}(m)$ with $2 \leq m \leq 8$.

Lemma 2.1. Let $G \in \mathbb{U}_{1}(m)$ with the unique cycle $C_{k}$ and $t$ pendent vertices, where $k+t=2 m, k \geq 3$ and $k \geq t \geq 1$.

(i) For $t=1,2,3, k-4, k-2, k,(k, t)=(10,4),(k, t)=(11,5)$, or $(k, t)=(12,4)$, we have

$$
K f(G) \geq \frac{1}{12}\left(k^{3}+2 k^{2} t+12 k t-k+2 t^{3}+12 t^{2}-16 t+\frac{t^{2}-t^{4}}{k}\right)
$$

with equality if and only if $G \cong U(k, t)$.

(ii) For integers $k, t$ with $k \geq 3, k \geq t \geq 1$, and $v \in V(G)$, we have

$$
K f_{G}(v) \geq f(k, t)
$$

with equality if and only if $G \cong U(k, t)$, and $v$ is a central vertex of the $t$ vertices of degree three in $U(k, t)$, where

$$
f(k, t)= \begin{cases}\frac{1}{12}\left(2 k^{2}+3 t^{2}+12 t-5-\frac{t^{3}-t}{k}\right) & \text { if } t \text { is odd } \\ \frac{1}{12}\left(2 k^{2}+3 t^{2}+12 t-2-\frac{t^{3}+2 t}{k}\right) & \text { if } t \text { is even } .\end{cases}
$$


Proof. First we prove (i). The cases $t=1, k-2, k$ are trivial. Suppose that $t \neq 1, k-2, k$.

Let $S(G)=\left\{v \in V\left(C_{k}\right): d_{G}(v)=3\right\}$, and let $\sigma(G)=\sum_{\left\{v_{i}, v_{j}\right\} \subseteq S(G)} r_{G}\left(v_{i}, v_{j}\right)$. Clearly, $|S(G)|=t$.

If $t=2$, say $S(G)=\left\{v_{1}, v_{s}\right\}$, then

$$
\sigma(G)=r_{G}\left(v_{1}, v_{s}\right) \geq \frac{1 \cdot(k-1)}{k}=\sigma(U(k, 2))
$$

with equality if and only if $v_{1}$ and $v_{s}$ are adjacent in $G$, i.e., $G \cong U(k, 2)$.

If $t \geq 3$, then by Eq. (1), we have

$$
\begin{aligned}
\sigma(U(k, t))= & r_{G}\left(v_{1}, v_{2}\right)+r_{G}\left(v_{1}, v_{3}\right)+\cdots+r_{G}\left(v_{1}, v_{t}\right) \\
& +r_{G}\left(v_{2}, v_{3}\right)+r_{G}\left(v_{2}, v_{4}\right)+\cdots+r_{G}\left(v_{2}, v_{t}\right) \\
& +\cdots+r_{G}\left(v_{t-1}, v_{t}\right) \\
= & \sum_{i=1}^{t-1} \sum_{j=i+1}^{t} r_{G}\left(v_{i}, v_{j}\right) \\
= & \sum_{i=1}^{t-1} \sum_{j=i+1}^{t} \frac{(j-i) \cdot[k-(j-i)]}{k} \\
= & \sum_{i=1}^{t-1} \sum_{j=1}^{t-i} \frac{j \cdot(k-j)}{k} \\
= & \sum_{i=1}^{t-1} \sum_{j=1}^{i} \frac{j \cdot(k-j)}{k} \\
= & \frac{1}{12 k} t(t-1)(t+1)(2 k-t) .
\end{aligned}
$$

Suppose that $t=3$. Then $k$ is odd as $G$ has perfect matching. By symmetry, we may assume that $S(G)=\left\{v_{1}, v_{i}, v_{j}\right\}$ with $1<i<j$, and $d_{G}\left(v_{1}, v_{i}\right) \leq d_{G}\left(v_{1}, v_{j}\right)$. Obviously, $i \leq \frac{k+1}{2}$. If $j \leq \frac{k+1}{2}$, then note that $d_{G}\left(v_{1}, v_{j}\right) \geq 2$, and by Eq. (1), we have

$$
\begin{aligned}
\sigma(G) & =r_{G}\left(v_{1}, v_{i}\right)+r_{G}\left(v_{1}, v_{j}\right)+r_{G}\left(v_{i}, v_{j}\right) \\
& \geq \frac{1 \cdot(k-1)}{k}+\frac{2 \cdot(k-2)}{k}+\frac{1 \cdot(k-1)}{k} \\
& =\sigma(U(k, 3))
\end{aligned}
$$

with equality if and only if $i=2$ and $j=3$, i.e., $G \cong U(k, 3)$. If $j=k$, then we have $i=2$ since $d_{G}\left(v_{1}, v_{i}\right) \leq d_{G}\left(v_{1}, v_{j}\right)$, i.e., $G \cong U(k, 3)$. Note that $j \neq k-1$ as $G$ has perfect matching. If $\frac{k+3}{2} \leq j \leq k-2$, then $d_{G}\left(v_{1}, v_{j}\right) \geq 3$, and by Eq. (1), we have

$$
\begin{aligned}
\sigma(G) & =r_{G}\left(v_{1}, v_{i}\right)+r_{G}\left(v_{1}, v_{j}\right)+r_{G}\left(v_{i}, v_{j}\right) \\
& \geq \frac{1 \cdot(k-1)}{k}+\frac{3 \cdot(k-3)}{k}+\frac{1 \cdot(k-1)}{k}
\end{aligned}
$$




$$
\begin{aligned}
& >\frac{1 \cdot(k-1)}{k}+\frac{2 \cdot(k-2)}{k}+\frac{1 \cdot(k-1)}{k} \\
& =\sigma(U(k, 3)) .
\end{aligned}
$$

Now it follows that $\sigma(G) \geq \sigma(U(k, 3))$ with equality if and only if $G \cong U(k, 3)$.

Suppose that $t=k-4 \geq 4$. Suppose to the contrary that $G ¥ U(k, k-4)$. Then there are two pairs of adjacent vertices of degree two on the cycle $C_{k}$ in $G$, separated by $a \geq 1$ consecutive vertices $v_{i_{1}}, v_{i_{2}}, \ldots, v_{i_{a}}$ of degree three and $b \geq 1$ consecutive vertices $v_{j_{1}}, v_{j_{2}}, \ldots, v_{j_{b}}$ of degree three on the cycle $C_{k}$, where $d_{C_{k}}\left(v_{i_{1}}, v_{j_{1}}\right)=3$, $d_{C_{k}}\left(v_{i_{a}}, v_{j_{b}}\right)=3$, and $a+b=k-4$. Assume that $a \geq b$. Denote by $w$ the pendent neighbor of $v_{j_{1}}$ in $G$. Consider $G^{\prime}=G-\left\{v_{j_{1}} w\right\}+\{v w\}$, where $v$ is the neighbor of $v_{i_{1}}$ with degree two on the cycle. Note that $S(G)=\left\{v_{i_{1}}, v_{i_{2}}, \ldots, v_{i_{a}}, v_{j_{1}}, v_{j_{2}}, \ldots, v_{j_{b}}\right\}$ and $S\left(G^{\prime}\right)=\left\{v_{i_{1}}, v_{i_{2}}, \ldots, v_{i_{a}}, v, v_{j_{2}}, \ldots, v_{j_{b}}\right\}$. If $b \geq 2$, then by Eq. (1), we have

$$
\begin{aligned}
& \sum_{s=1}^{a} r_{G}\left(v_{j_{1}}, v_{i_{s}}\right)-\sum_{s=1}^{a} r_{G^{\prime}}\left(v, v_{i_{s}}\right)=\frac{1}{k} \sum_{i=3}^{a+2} i(k-i)-\frac{1}{k} \sum_{i=1}^{a} i(k-i), \\
& \sum_{s=2}^{b} r_{G}\left(v_{j_{1}}, v_{j_{s}}\right)-\sum_{s=2}^{b} r_{G^{\prime}}\left(v, v_{j_{s}}\right)=\frac{1}{k} \sum_{i=1}^{b-1} i(k-i)-\frac{1}{k} \sum_{i=3}^{b+1} i(k-i),
\end{aligned}
$$

and thus

$$
\begin{aligned}
= & \sum_{x \in S(G) \backslash\left\{v_{j_{1}}\right\}} r_{G}\left(v_{j_{1}}, x\right)-\sum_{x \in S\left(G^{\prime}\right) \backslash\{v\}} r_{G^{\prime}}(v, x) \\
= & \left(\sum_{s=1}^{a} r_{G}\left(v_{j_{1}}, v_{i_{s}}\right)+\sum_{s=2}^{b} r_{G}\left(v_{j_{1}}, v_{j_{s}}\right)\right) \\
& -\left(\sum_{s=1}^{a} r_{G^{\prime}}\left(v, v_{i_{s}}\right)+\sum_{s=2}^{b} r_{G^{\prime}}\left(v, v_{j_{s}}\right)\right) \\
= & \left(\sum_{s=1}^{a} r_{G}\left(v_{j_{1}}, v_{i_{s}}\right)-\sum_{s=1}^{a} r_{G^{\prime}}\left(v, v_{i_{s}}\right)\right) \\
& +\left(\sum_{s=2}^{b} r_{G}\left(v_{j_{1}}, v_{j_{s}}\right)-\sum_{s=2}^{b} r_{G^{\prime}}\left(v, v_{j_{s}}\right)\right) \\
= & \left(\frac{1}{k} \sum_{i=3}^{a+2} i(k-i)-\frac{1}{k} \sum_{i=1}^{a} i(k-i)\right) \\
& +\left(\frac{1}{k} \sum_{i=1}^{b-1} i(k-i)-\frac{1}{k} \sum_{i=3}^{b+1} i(k-i)\right)
\end{aligned}
$$




$$
=\frac{4}{k}(a-b+1)>0
$$

If $b=1$, then by similar arguments as above, we have $\sigma(G)-\sigma\left(G^{\prime}\right)=\frac{4 a}{k}>0$. Thus $\sigma(G)>\sigma\left(G^{\prime}\right)$ for $b \geq 1$. By repeating the transformation from $G$ to $G^{\prime}$, we may finally get $\sigma(G)>\sigma(U(k, k-4))$. Thus if $t=k-4$, then $\sigma(G) \geq \sigma(U(k, k-4))$ with equality if and only if $G \cong U(k, k-4)$.

Suppose that $(k, t)=(10,4)$. Then there are exactly four possibilities for $G$, and by suitable labeling, we may assume that $S(G)=\left\{v_{1}, v_{2}, v_{3}, v_{4}\right\},\left\{v_{1}, v_{2}, v_{3}, v_{6}\right\},\left\{v_{1}, v_{2}, v_{5}, v_{6}\right\}$, or $\left\{v_{1}, v_{2}, v_{5}, v_{8}\right\}$. By direct calculation, we have

$$
\sigma(G)= \begin{cases}8 & \text { if } S(G)=\left\{v_{1}, v_{2}, v_{3}, v_{4}\right\} \\ \frac{52}{5} & \text { if } S(G)=\left\{v_{1}, v_{2}, v_{3}, v_{6}\right\} \\ \frac{56}{5} & \text { if } S(G)=\left\{v_{1}, v_{2}, v_{5}, v_{6}\right\} \\ 12 & \text { if } S(G)=\left\{v_{1}, v_{2}, v_{5}, v_{8}\right\}\end{cases}
$$

and thus $\sigma(G) \geq 8$ with equality if and only if $G \cong U(10,4)$.

Suppose that $(k, t)=(11,5)$. Then there are exactly five possibilities for $G$, and by suitable labeling, we may assume that $S(G)=\left\{v_{1}, v_{2}, v_{3}, v_{4}, v_{5}\right\},\left\{v_{1}, v_{2}, v_{3}, v_{4}, v_{7}\right\}$, $\left\{v_{1}, v_{2}, v_{3}, v_{6}, v_{7}\right\},\left\{v_{1}, v_{2}, v_{3}, v_{6}, v_{9}\right\}$, or $\left\{v_{1}, v_{2}, v_{5}, v_{8}, v_{9}\right\}$. By direct calculation, we have

$$
\sigma(G)= \begin{cases}\frac{170}{11} & \text { if } S(G)=\left\{v_{1}, v_{2}, v_{3}, v_{4}, v_{5}\right\}, \\ \frac{202}{11} & \text { if } S(G)=\left\{v_{1}, v_{2}, v_{3}, v_{4}, v_{7}\right\}, \\ \frac{218}{11} & \text { if } S(G)=\left\{v_{1}, v_{2}, v_{3}, v_{6}, v_{7}\right\}, \\ \frac{226}{11} & \text { if } S(G)=\left\{v_{1}, v_{2}, v_{3}, v_{6}, v_{9}\right\}, \\ \frac{234}{11} & \text { if } S(G)=\left\{v_{1}, v_{2}, v_{5}, v_{8}, v_{9}\right\}\end{cases}
$$

and thus $\sigma(G) \geq \frac{170}{11}$ with equality if and only if $G \cong U(11,5)$.

Suppose that $(k, t)=(12,4)$. Then there are exactly eight possibilities for $G$, and by suitable labeling, we may assume that $S(G)=\left\{v_{1}, v_{2}, v_{3}, v_{4}\right\},\left\{v_{1}, v_{2}, v_{3}, v_{6}\right\},\left\{v_{1}, v_{2}, v_{3}, v_{8}\right\}$, $\left\{v_{1}, v_{2}, v_{5}, v_{6}\right\},\left\{v_{1}, v_{2}, v_{7}, v_{8}\right\},\left\{v_{1}, v_{2}, v_{5}, v_{8}\right\},\left\{v_{1}, v_{2}, v_{5}, v_{10}\right\}$, or $\left\{v_{1}, v_{4}, v_{7}, v_{10}\right\}$. By direct calculation, we have

$$
\sigma(G)= \begin{cases}\frac{25}{3} & \text { if } S(G)=\left\{v_{1}, v_{2}, v_{3}, v_{4}\right\}, \\ \frac{34}{3} & \text { if } S(G)=\left\{v_{1}, v_{2}, v_{3}, v_{6}\right\}, \\ \frac{37}{3} & \text { if } S(G)=\left\{v_{1}, v_{2}, v_{3}, v_{8}\right\}, \\ \frac{37}{3} & \text { if } S(G)=\left\{v_{1}, v_{2}, v_{5}, v_{6}\right\}, \\ 14 & \text { if } S(G)=\left\{v_{1}, v_{2}, v_{7}, v_{8}\right\}, \\ \frac{41}{3} & \text { if } S(G)=\left\{v_{1}, v_{2}, v_{5}, v_{8}\right\}, \\ 15 & \text { if } S(G)=\left\{v_{1}, v_{4}, v_{7}, v_{10}\right\},\end{cases}
$$


and thus $\sigma(G) \geq \frac{25}{3}$ with equality if and only if $G \cong U(12,4)$.

Combining all the above cases, and by Eq. (4), we can deduce that

$$
\sigma(G) \geq \sigma(U(k, t))=\frac{1}{12 k} t(t-1)(t+1)(2 k-t)
$$

with equality if and only if $G \cong U(k, t)$ for $t=1,2,3, k-4, k-2, k,(k, t)=(10,4)$, $(k, t)=(11,5)$, or $(k, t)=(12,4)$. For $1 \leq i \leq k$ with $d_{G}\left(v_{i}\right)=3$, let $u_{i}$ be the pendent neighbor of $v_{i}$ in $G$.

By Eqs. (3) and (2), we have

$$
\begin{aligned}
K f(G)= & \sum_{\left\{v_{i}, v_{j}\right\} \subseteq V\left(C_{k}\right)} r_{G}\left(v_{i}, v_{j}\right)+\sum_{u_{i} \in V(G) \backslash V\left(C_{k}\right)} \sum_{v_{j} \in V\left(C_{k}\right)} r_{G}\left(u_{i}, v_{j}\right) \\
& +\sum_{\left\{u_{i}, u_{j}\right\} \subseteq V(G) \backslash V\left(C_{k}\right)} r_{G}\left(u_{i}, u_{j}\right) \\
= & \frac{k^{3}-k}{12}+\sum_{v_{i} \in S(G)} \sum_{v_{j} \in V\left(C_{k}\right)}\left(1+r_{G}\left(v_{i}, v_{j}\right)\right)+\sum_{\left\{v_{i}, v_{j}\right\} \subseteq S(G)}\left(2+r_{G}\left(v_{i}, v_{j}\right)\right) \\
= & \frac{k^{3}-k}{12}+\sum_{v_{i} \in S(G)}\left(k+K f_{C_{k}}\left(v_{i}\right)\right)+2\left(\begin{array}{l}
t \\
2
\end{array}\right)+\sum_{\left\{v_{i}, v_{j}\right\} \subseteq S(G)} r_{G}\left(v_{i}, v_{j}\right) \\
= & \frac{k^{3}-k}{12}+t\left(k+\frac{k^{2}-1}{6}\right)+2\left(\begin{array}{l}
t \\
2
\end{array}\right)+\sigma(G) \\
\geq & \frac{k^{3}-k}{12}+t\left(k+\frac{k^{2}-1}{6}\right)+2\left(\begin{array}{l}
t \\
2
\end{array}\right)+\frac{1}{12 k} t(t-1)(t+1)(2 k-t) \\
= & \frac{1}{12}\left(k^{3}+2 k^{2} t+12 k t-k+2 t^{3}+12 t^{2}-16 t+\frac{t^{2}-t^{4}}{k}\right)
\end{aligned}
$$

with equality if and only if $G \cong U(k, t)$ for $t=1,2,3, k-4, k-2, k,(k, t)=(10,4)$, $(k, t)=(11,5)$, or $(k, t)=(12,4)$.

Next we prove (ii). Let $v \in V(G)$. For $v_{i} \in V\left(C_{k}\right)$, clearly $K f_{G}\left(v_{i}^{*}\right)-K f_{G}\left(v_{i}\right)=$ $2 m-2>0$, where $d_{G}\left(v_{i}\right)=3$, and $v_{i}^{*}$ is the unique neighbor of $v_{i}$ in $G$ outside $C_{k}$. Thus we may assume that $v=v_{i} \in V\left(C_{k}\right)$. By Eq. (1), it is easily seen that

$$
\begin{aligned}
\sum_{v_{j} \in S(G)} r_{G}\left(v_{i}, v_{j}\right) & \geq \begin{cases}0+2 \sum_{i=1}^{(t-1) / 2} \frac{i \cdot(k-i)}{k} & \text { if } t \text { is odd } \\
0+2 \sum_{i=1}^{(t-2) / 2} \frac{i \cdot(k-i)}{k}+\frac{t / 2 \cdot(k-t / 2)}{k} & \text { if } t \text { is even }\end{cases} \\
& = \begin{cases}\frac{1}{12}\left(3 t^{2}-3-\frac{t^{3}-t}{k}\right) & \text { if } t \text { is odd } \\
\frac{1}{12}\left(3 t^{2}-\frac{t^{3}+2 t}{k}\right) & \text { if } t \text { is even }\end{cases}
\end{aligned}
$$

with equality if and only if the $t$ vertices in $S(G)$ are consecutive on $C_{k}$, i.e., $G \cong U(k, t)$, and $v_{i}$ is a central vertex of the $t$ vertices of degree three in $U(k, t)$. For $v_{i} \in V\left(C_{k}\right)$, by 
Eq. (2), we have

$$
\begin{aligned}
K f_{G}\left(v_{i}\right) & =K f_{C_{k}}\left(v_{i}\right)+\sum_{u_{j} \in V(G) \backslash V\left(C_{k}\right)} r_{G}\left(v_{i}, u_{j}\right) \\
& =\frac{k^{2}-1}{6}+\sum_{v_{j} \in S(G)}\left(1+r_{G}\left(v_{i}, v_{j}\right)\right) \\
& =\frac{k^{2}-1}{6}+t+\sum_{v_{j} \in S(G)} r_{G}\left(v_{i}, v_{j}\right) \\
& \geq \begin{cases}\frac{k^{2}-1}{6}+t+\frac{1}{12}\left(3 t^{2}-3-\frac{t^{3}-t}{k}\right) & \text { if } t \text { is odd } \\
\frac{k^{2}-1}{6}+t+\frac{1}{12}\left(3 t^{2}-\frac{t^{3}+2 t}{k}\right) & \text { if } t \text { is even }\end{cases} \\
& = \begin{cases}\frac{1}{12}\left(2 k^{2}+3 t^{2}+12 t-5-\frac{t^{3}-t}{k}\right) & \text { if } t \text { is odd } \\
\frac{1}{12}\left(2 k^{2}+3 t^{2}+12 t-2-\frac{t^{3}+2 t}{k}\right) & \text { if } t \text { is even }\end{cases} \\
& =f(k, t)
\end{aligned}
$$

with equality if and only if $G \cong U(k, t)$, and $v_{i}$ is a central vertex of the $t$ vertices of degree three in $U(k, t)$.

If $G \in \mathbb{U}_{1}(m)$ with the unique cycle $C_{k}$ and $t$ pendent vertices, where $2 \leq m \leq 8$, then $t=1,2,3, k-4, k-2, k,(k, t)=(10,4),(k, t)=(11,5)$, or $(k, t)=(12,4)$. Now by Lemma 2.1 (i), we have

Lemma 2.2. If $G$ is a graph in $\mathbb{U}_{1}(m)$ with the minimum Kirchhoff index, where $2 \leq$ $m \leq 8$, then $G \cong U(k, t)$ with $k+t=2 m, k \geq 3$ and $k \geq t \geq 1$.

\subsection{The Kirchhoff index of graphs in $\mathbb{U}_{2}(m)$ with small $m$}

The following result will be useful for comparing the Kirchhoff indices of graphs.

For simplicity, let $|G|=|V(G)|$ for a graph $G$.

Lemma 2.3. [12] Let $G$ and $H$ be two connected graphs with $u \in V(G)$ and $w \in V(H)$. Let $G u H$ be the graph obtained from $G$ and $H$ by identifying $u \in V(G)$ with $w \in V(H)$. Then

$$
K f(G u H)=K f(G)+K f(H)+(|H|-1) K f_{G}(u)+(|G|-1) K f_{H}(w) .
$$

Let $P_{n}$ be the path on $n$ vertices.

If $u$ is a pendent vertex being adjacent to a vertex $v$ of degree two in the graph $G$, then the path of $G$ induced by the vertices $u$ and $v$ is said to be a pendent $P_{2}$ of $G$. Clearly, every graph in $\mathbb{U}_{2}(m)$ has at least one pendent $P_{2}$.

For a given graph $G \in \mathbb{U}_{2}(m)$, starting from $G$, deleting the pendent $P_{2}$ 's repeatedly, until there is no pendent $P_{2}$, the resulting graph is denoted by $\bar{G}$. Let $\bar{n}=|\bar{G}|$. Clearly, $\bar{G} \in \mathbb{U}_{1}\left(\frac{\bar{n}}{2}\right) \cup\left\{C_{\bar{n}}\right\}$. 
Now we determine the minimum Kirchhoff index among the graphs in $\mathbb{U}_{2}(m)$ with $3 \leq m \leq 8$.

Lemma 2.4. If $G$ is a graph in $\mathbb{U}_{2}(m)$ with the minimum Kirchhoff index, where $3 \leq$ $m \leq 8$, then $G \cong U(k, t, 0, j)$ with $k+t+2 j=2 m, k \geq 3, k \geq t \geq 1$ and $j \geq 1$.

Proof. Let $G \in \mathbb{U}_{2}(m)$, and $k$ be the length of the unique cycle of $G$.

Denote by the deleting process from $G$ to $\bar{G}$ as follows:

$$
G=G_{1} \rightarrow G_{2} \rightarrow \cdots \rightarrow G_{r-1} \rightarrow G_{r}=\bar{G},
$$

where $G_{i+1}$ is the (unicyclic) graph obtained from $G_{i}$ by deleting a pendent $P_{2}$, where $1 \leq i \leq r-1$. Note that $\bar{n}+2(r-1)=2 m$.

Recall that $G_{r}=\bar{G} \in \mathbb{U}_{1}\left(\frac{\bar{n}}{2}\right) \cup\left\{C_{\bar{n}}\right\}$. By Lemma 2.2, we have $K f\left(G_{r}\right) \geq K f(U(k, t, 0,0))$, where $k+t=\bar{n}$. Moreover, by Lemma 2.1 (ii) and Lemma 2.3, we have $K f\left(G_{r-1}\right) \geq$ $K f(U(k, t, 0,1))$ with equality if and only if $G_{r-1} \cong U(k, t, 0,1)$. Again by Lemma 2.1 (ii) and Lemma 2.3, we have $K f\left(G_{r-2}\right) \geq K f(U(k, t, 0,2))$ with equality if and only if $G_{r-2} \cong U(k, t, 0,2)$. Repeating the arguments, finally we can deduce that $K f(G)=$ $K f\left(G_{1}\right) \geq K f(U(k, t, 0, r-1))$ with last equality if and only if $G \cong U(k, t, 0, r-1)$.

Then the result follows easily.

\subsection{The effect on the Kirchhoff index of graphs under the dele- tion of some vertices}

First we introduce a unicyclic graph.

Let $U_{n, m}=U(5,1, n-2 m, m-3)$, where $3 \leq m \leq\left\lfloor\frac{n}{2}\right\rfloor$, see Fig 2. It is easily checked that

$$
K f\left(U_{n, m}\right)=n^{2}+n m-5 n-3 m+4 .
$$

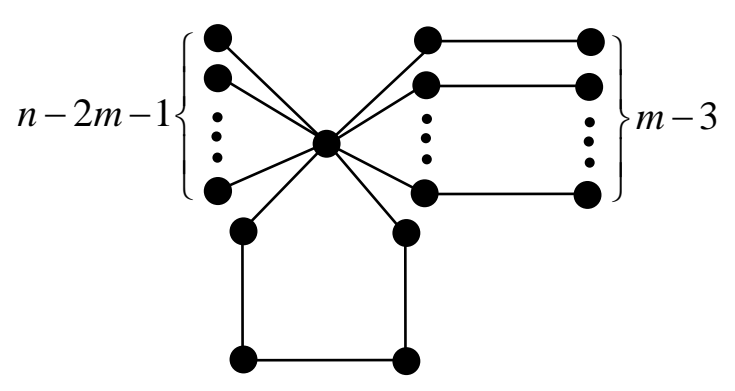

Figure 2: The graph $U_{n, m}$.

Next we establish a lower bound of $K f_{G}(u)$, where $G \in \mathbb{U}(n, m)$ and $u \in V(G)$. 
Lemma 2.5. Let $G \in \mathbb{U}(n, m)$ with the unique cycle $C_{k}$, where $n \geq 6, m \geq 3, k \geq 3$. If $T_{i} \cong P_{1}$ or $P_{2}$ for $2 \leq i \leq k$, then for $u \in V\left(T_{1}\right)$,

$$
K f_{G}(u) \geq n+m-4
$$

with equality if and only if $G \cong U_{n, m}$, and $u$ is the vertex of maximum degree in $U_{n, m}$.

Proof. Let $M$ be a maximum matching of $G$. First we establish an upper bound of $d_{G}(u)$. Let

$$
\begin{gathered}
A_{1}=\{x y \in E(G) \backslash M: \text { either } x=u \text { or } y=u\}, \\
A_{2}=\left\{x y \in E(G) \backslash M: x, y \neq u \text { and } x y \in E\left(C_{k}\right)\right\}, \\
A_{3}=\left\{x y \in E(G) \backslash M: x, y \neq u \text { and } x y \notin E\left(C_{k}\right)\right\} .
\end{gathered}
$$

Clearly, $A_{1}, A_{2}, A_{3}$ are pairwise disjoint, and $E(G) \backslash M=A_{1} \cup A_{2} \cup A_{3}$. Thus

$$
|E(G) \backslash M|=n-m=\left|A_{1}\right|+\left|A_{2}\right|+\left|A_{3}\right| .
$$

Note that

(a) $\left|A_{1}\right| \geq d_{G}(u)-1$ with equality if and only if $u$ is $M$-saturated;

(b) $\left|A_{2}\right| \geq\left\lfloor\frac{k-2}{2}\right\rfloor$ if $u$ lies on the unique cycle $C_{k}$ of $G$, and $\left|A_{2}\right| \geq\left\lfloor\frac{k+1}{2}\right\rfloor$ if $u$ lies outside the unique cycle $C_{k}$ of $G$;

(c) $\left|A_{3}\right| \geq 0$.

It follows from Eq. (6) that if $u$ lies on the unique cycle $C_{k}$ of $G$, then $n-m \geq\left(d_{G}(u)-\right.$ 1) $+\left\lfloor\frac{k-2}{2}\right\rfloor$, i.e.,

$$
d_{G}(u) \leq n-m+1-\left\lfloor\frac{k-2}{2}\right\rfloor
$$

with equality if and only if the corresponding equalities in (a), (b), (c) hold, while if $u$ lies outside the unique cycle $C_{k}$ of $G$, then $n-m \geq\left(d_{G}(u)-1\right)+\left\lfloor\frac{k+1}{2}\right\rfloor$, i.e.,

$$
d_{G}(u) \leq n-m+1-\left\lfloor\frac{k+1}{2}\right\rfloor
$$

with equality if and only if the corresponding equalities in (a), (b), (c) hold.

Case 1. $u$ lies on the unique cycle $C_{k}$ of $G$.

Subcase 1.1. $k$ is odd and $T_{i} \cong P_{1}$ for $2 \leq i \leq k$.

By Eq. (2) and inequality (7), we have

$$
K f_{G}(u)=K f_{C_{k}}(u)+\sum_{x \in V(G) \backslash V\left(C_{k}\right)} r_{G}(u, x)
$$




$$
\begin{aligned}
& \geq \frac{k^{2}-1}{6}+\left[\left(d_{G}(u)-2\right)+2\left(n-k-d_{G}(u)+2\right)\right] \\
& =-d_{G}(u)+\frac{1}{6} k^{2}-2 k+2 n+\frac{11}{6} \\
& \geq-\left(n-m+1-\frac{k-3}{2}\right)+\frac{1}{6} k^{2}-2 k+2 n+\frac{11}{6} \\
& =\frac{1}{6}\left(k^{2}-9 k+6 n+6 m-4\right)
\end{aligned}
$$

with equality if and only if $G \cong U\left(k, 1, n-2 m, m-\frac{k+1}{2}\right)$ with odd $k$, and $u$ is the vertex of maximum degree in $U\left(k, 1, n-2 m, m-\frac{k+1}{2}\right)$.

Subcase 1.2. $k$ is even and $T_{i} \cong P_{1}$ for $2 \leq i \leq k$, or there is at least one of $T_{i}$ such that $T_{i} \cong P_{2}$ for $2 \leq i \leq k$.

Obviously, $r_{G}(u, v) \geq 1+\frac{2 \cdot(k-2)}{k} \geq 2$, where $v$ is the unique pendent neighbor of $v_{i}$ with $3 \leq i \leq k-1$ if $T_{i} \cong P_{2}$. On the other hand, we also note that if $k$ is odd, then $T_{i} \cong P_{2}$ for some $i$ with $2 \leq i \leq k$, and thus either $\left|A_{2}\right|>\left\lfloor\frac{k-2}{2}\right\rfloor=\frac{k-3}{2}$ or $\left|A_{3}\right|>0$, by Eq. (6), we have $n-m \geq\left(d_{G}(u)-1\right)+\frac{k-3}{2}+1$, i.e.,

$$
d_{G}(u) \leq n-m-\frac{k-3}{2} .
$$

Let $a$ be the number of pendent vertices attached to $v_{2}$ or $v_{k}$ in $G$, where $0 \leq a \leq 2$. Then by Eq. (2), and inequalities (7) and (9), we have

$$
\begin{aligned}
K f_{G}(u) & =K f_{C_{k}}(u)+\sum_{x \in V(G) \backslash V\left(C_{k}\right)} r_{G}(u, x) \\
& \geq \frac{k^{2}-1}{6}+\left[\left(d_{G}(u)-2\right)+\left(1+\frac{1 \cdot(k-1)}{k}\right) a+2\left(n-k-a-d_{G}(u)+2\right)\right] \\
& =-\frac{a}{k}-d_{G}(u)+\frac{1}{6} k^{2}-2 k+2 n+\frac{11}{6} \\
& \geq \begin{cases}-\frac{2}{k}-\left(n-m+1-\frac{k-2}{2}\right)+\frac{1}{6} k^{2}-2 k+2 n+\frac{11}{6} & \text { if } k \text { is even } \\
-\frac{2}{k}-\left(n-m-\frac{k-3}{2}\right)+\frac{1}{6} k^{2}-2 k+2 n+\frac{11}{6} & \text { if } k \text { is odd }\end{cases} \\
& = \begin{cases}\frac{1}{6}\left(k^{2}-9 k+6 n+6 m-1-\frac{12}{k}\right) & \text { if } k \text { is even } \\
\frac{1}{6}\left(k^{2}-9 k+6 n+6 m+2-\frac{12}{k}\right) & \text { if } k \text { is odd } \\
\geq & \frac{1}{6}\left(k^{2}-9 k+6 n+6 m-4\right) .\end{cases}
\end{aligned}
$$

If $K f_{G}(u)=\frac{1}{6}\left(k^{2}-9 k+6 n+6 m-4\right)$, then $a=2, k=4$ and $d_{G}(u)=n-m+1-\frac{k-2}{2}=n-m$. However, $a=2$ and $k=4$ imply that either $\left|A_{2}\right|>1$ or $\left|A_{3}\right|>0$, and thus by Eq. (6), we have $n-m>d_{G}(u)$. Therefore $K f_{G}(u)>\frac{1}{6}\left(k^{2}-9 k+6 n+6 m-4\right)$.

Case 2. $u$ lies outside the unique cycle $C_{k}$ of $G$. 
Note that for $v \notin V\left(C_{k}\right) \cup V\left(T_{1}\right), r_{G}(u, v) \geq 2+\frac{1 \cdot(k-1)}{k}>2$. Let $b$ be the number of neighbors of $u$ on $C_{k}$, where $b=0,1$. Now by Eq. (2) and inequality (8), we have

$$
\begin{aligned}
K f_{G}(u) & =K f_{C_{k}}(u)+\sum_{x \in V(G) \backslash V\left(C_{k}\right)} r_{G}(u, x) \\
& \geq\left(k+\frac{k^{2}-1}{6}\right)+\left[\left(d_{G}(u)-b\right)+2\left(n-k-d_{G}(u)+b\right)\right] \\
& =b-d_{G}(u)+\frac{1}{6} k^{2}-k+2 n-\frac{1}{6} \\
& \geq 0-\left(n-m+1-\left\lfloor\frac{k+1}{2}\right\rfloor\right)+\frac{1}{6} k^{2}-k+2 n-\frac{1}{6} \\
& >\frac{1}{6}\left(k^{2}-9 k+6 n+6 m-4\right) .
\end{aligned}
$$

Now combining Cases 1 and 2, we have

$$
K f_{G}(u) \geq \frac{1}{6}\left(k^{2}-9 k+6 n+6 m-4\right)
$$

with equality if and only if $G \cong U\left(k, 1, n-2 m, m-\frac{k+1}{2}\right)$ with odd $k$, and $u$ is the vertex of maximum degree in $U\left(k, 1, n-2 m, m-\frac{k+1}{2}\right)$. Thus

$$
\begin{aligned}
K f_{G}(u) \geq \frac{1}{6}\left(k^{2}-9 k+6 n+6 m-4\right) & \geq \frac{1}{6}\left(5^{2}-9 \cdot 5+6 n+6 m-4\right) \\
& =n+m-4
\end{aligned}
$$

with equalities if and only if $G \cong U(5,1, n-2 m, m-3)=U_{n, m}$, and $u$ is the vertex of maximum degree in $U_{n, m}$.

Now we present a stronger version of lemma 2.5.

Lemma 2.6. Let $G$ be a unicyclic graph with $n$ vertices and matching number at least $m$, where $n \geq 6, m \geq 3$. For $u \in V(G)$,

$$
K f_{G}(u) \geq n+m-4
$$

with equality if and only if $G \cong U_{n, m}$, and $u$ is the vertex of maximum degree in $U_{n, m}$.

Proof. Let $G$ be a unicyclic graph with a vertex $u \in V(G)$ such that

$$
K f_{G}(u)=\min \left\{K f_{H}(x): H \in \mathbb{U}(n, r), x \in V(H), r \geq m\right\} .
$$

Assume that $u \in V\left(T_{1}\right)$. Let $M$ be a maximum matching of $G$. Suppose that $\left|T_{i}\right| \geq 3$ for some $i$ with $2 \leq i \leq k$, where $k$ is the length of the unique cycle of $G$. Then there is some edge, say $x y$, in $T_{i}$ outside $M$. Assume that the vertices $x$ and $u$ lie in the same component of $G-x y$. Let $G_{1}=G-x y+u y$. Clearly, $M$ is also a matching of $G_{1}$, 
and thus $G_{1}$ has matching number at least $m$. However, $K f_{G_{1}}(u)<K f_{G}(u)$, which is a contradiction. Thus $\left|T_{i}\right|=1,2$, i.e., $T_{i} \cong P_{1}$ or $P_{2}$ for $2 \leq i \leq k$. By Lemma 2.5, we have

$$
K f_{G}(u) \geq n+r-4 \geq n+m-4
$$

with equalities if and only if $G \cong U_{n, m}$, and $u$ is the vertex of maximum degree in $U_{n, m}$.

The following result turns out to be of rather important for the proof of our main results.

Lemma 2.7. Let $G \in \mathbb{U}(n, m)$ with a pendent vertex $x$ being adjacent to vertex $y$, and let $z$ be the neighbor of $y$ different from $x$ if $d_{G}(y)=2$, where $n \geq 6, m \geq 3$. Then

$$
K f(G)-K f(G-x) \geq 2 n+m-6
$$

with equality if and only if $G \cong U_{n, m}$, and $x$ is a pendent neighbor of the vertex of maximum degree in $U_{n, m}$. Moreover, if $d_{G}(y)=2$, then

$$
K f(G)-K f(G-x-y) \geq 5 n+2 m-19
$$

with equality if and only if $G \cong U_{n, m}$.

Proof. Note that $K f_{G}(x)-K f_{G}(y)=n-2$. Then by Lemma 2.6, we have

$$
\begin{aligned}
K f(G)-K f(G-x) & =K f_{G}(x) \\
& =K f_{G}(y)+n-2 \\
& \geq(n+m-4)+n-2=2 n+m-6
\end{aligned}
$$

with equality if and only if $G \cong U_{n, m}$, and $x$ is a pendent neighbor of the vertex of maximum degree in $U_{n, m}$.

If $d_{G}(y)=2$, then $K f_{G}(y)-K f_{G}(z)=n-4$, and thus by Lemma 2.6, we have

$$
\begin{aligned}
K f(G)-K f(G-x-y) & =K f_{G}(x)+K f_{G}(y)-1 \\
& =2 K f_{G}(z)+3 n-11 \\
& \geq 2(n+m-4)+3 n-11=5 n+2 m-19
\end{aligned}
$$

with equality if and only if $G \cong U_{n, m}$.

Lemma 2.8. [1] Let $G$ be an n-vertex unicyclic graph with the unique cycle $C_{k}$, where $3 \leq k \leq n-1$. Then

$$
K f(G) \geq \frac{1}{12}\left[-k^{3}+2 n k^{2}-(12 n-13) k+12 n^{2}-14 n\right]
$$

with equality if and only if $G \cong U(k, 1, n-k-1,0)$. 


\section{Results}

First we consider the minimum Kirchhoff index of unicyclic graphs with perfect matching.

Theorem 3.1. Among the graphs in $\mathbb{U}(2 m, m)$ with $m \geq 2, C_{2 m}$ for $2 \leq m \leq 4, U(8,2)$ for $m=5, U(8,4)$ for $m=6, U(7,7)$ for $m=7$, and $U_{2 m, m}$ for $m \geq 8$ are the unique graphs with the minimum Kirchhoff indices, which are equal to $\frac{1}{6}\left(4 m^{3}-m\right)$ for $2 \leq m \leq 4$, $81 \frac{7}{8}$ for $m=5,135 \frac{1}{2}$ for $m=6,203$ for $m=7$, and $6 m^{2}-13 m+4$ for $m \geq 8$.

Proof. Recall that $\mathbb{U}(2 m, m)=\mathbb{U}_{1}(m) \cup \mathbb{U}_{2}(m) \cup\left\{C_{2 m}\right\}$.

Case 1. $2 \leq m \leq 8$.

The case $m=2$ is obvious since $\mathbb{U}(4,2)=\left\{U(3,1), C_{4}\right\}$, where

$$
K f(U(3,1))=6 \frac{1}{3}>5=K f\left(C_{4}\right) .
$$

For $3 \leq m \leq 8$, by Lemmas 2.2 and 2.4, the minimum Kirchhoff index of the graphs in $\mathbb{U}(2 m, m)$ is precisely achieved by some graph of the form $U(k, t, 0, j)$, where $k+t+2 j=$ $2 m, k \geq 3, k \geq t \geq 0$ and $j \geq 0$. In Tables $1-6$ corresponding to $m=3,4, \ldots, 8$, we list these graphs and their Kirchhoff indices. We use $(k, t ; j)$ to represent the graph $U(k, t, 0, j)$ in these tables. From these tables, we find that

(1) $U(6,0,0,0)=C_{6}$ is the unique graph in $\mathbb{U}(6,3)$ with the minimum Kirchhoff index, which is equal to $17 \frac{1}{2}$;

(2) $U(8,0,0,0)=C_{8}$ is the unique graph in $\mathbb{U}(8,4)$ with the minimum Kirchhoff index, which is equal to 42 ;

(3) $U(8,2,0,0)=U(8,2)$ is the unique graph in $\mathbb{U}(10,5)$ with the minimum Kirchhoff index, which is equal to $81 \frac{7}{8}$;

(4) $U(8,4,0,0)=U(8,4)$ is the unique graph in $\mathbb{U}(12,6)$ with the minimum Kirchhoff index, which is equal to $135 \frac{1}{2}$;

(5) $U(7,7,0,0)=U(7,7)$ is the unique graph in $\mathbb{U}(14,7)$ with the minimum Kirchhoff index, which is equal to 203 ;

(6) $U(5,1,0,5)=U_{16,8}$ is the unique graph in $\mathbb{U}(16,8)$ with the minimum Kirchhoff index, which is equal to 284 .

Case 2. $m \geq 9$.

We prove the result by induction on $m$. Suppose that the result holds for all the graphs in $\mathbb{U}(2 m-2, m-1)$. Let $G \in \mathbb{U}(2 m, m)$.

If $G \cong C_{2 m}$, then by Eqs. (3) and (5), we have

$$
K f(G)=\frac{1}{6}\left(4 m^{3}-m\right)>6 m^{2}-13 m+4=K f\left(U_{2 m, m}\right) .
$$


Table 1: The Kirchhoff indices of the graphs $U(k, t, 0, j)$ in $\mathbb{U}(6,3)$.

\begin{tabular}{|c||c|c|c|c|c|c|}
\hline Graphs & $(3,1 ; 1)$ & $(3,3 ; 0)$ & $(4,0 ; 1)$ & $(4,2 ; 0)$ & $(5,1 ; 0)$ & $(6,0 ; 0)$ \\
\hline Kirchhoff indices & 24 & 23 & 23 & $20 \frac{3}{4}$ & 19 & $17 \frac{1}{2}$ \\
\hline
\end{tabular}

Table 2: The Kirchhoff indices of the graphs $U(k, t, 0, j)$ in $\mathbb{U}(8,4)$.

\begin{tabular}{|c||c|c|c|c|c|c|}
\hline Graphs & $(3,1 ; 2)$ & $(3,3 ; 1)$ & $(4,0 ; 2)$ & $(4,2 ; 1)$ & $(4,4 ; 0)$ & $(5,1 ; 1)$ \\
\hline Kirchhoff indices & $53 \frac{2}{3}$ & $53 \frac{1}{3}$ & 53 & $50 \frac{1}{4}$ & 48 & 48 \\
\hline Graphs & $(5,3 ; 0)$ & $(6,0 ; 1)$ & $(6,2 ; 0)$ & $(7,1 ; 0)$ & $(8,0 ; 0)$ & \\
\hline Kirchhoff indices & $45 \frac{4}{5}$ & $48 \frac{1}{6}$ & 44 & 43 & 42 & \\
\hline
\end{tabular}

Table 3: The Kirchhoff indices of the graphs $U(k, t, 0, j)$ in $\mathbb{U}(10,5)$.

\begin{tabular}{|c||c|c|c|c|c|c|}
\hline Graphs & $(3,1 ; 3)$ & $(3,3 ; 2)$ & $(4,0 ; 3)$ & $(4,2 ; 2)$ & $(4,4 ; 1)$ & $(5,1 ; 2)$ \\
\hline Kirchhoff indices & $95 \frac{1}{3}$ & $95 \frac{2}{3}$ & 95 & $91 \frac{3}{4}$ & 91 & 89 \\
\hline Graphs & $(5,3 ; 1)$ & $(5,5 ; 0)$ & $(6,0 ; 2)$ & $(6,2 ; 1)$ & $(6,4 ; 0)$ & $(7,1 ; 1)$ \\
\hline Kirchhoff indices & 88 & 85 & $90 \frac{5}{6}$ & $86 \frac{1}{3}$ & $83 \frac{1}{2}$ & 86 \\
\hline Graphs & $(7,3 ; 0)$ & $(8,0 ; 1)$ & $(8,2 ; 0)$ & $(9,1 ; 0)$ & $(10,0 ; 0)$ & \\
\hline Kirchhoff indices & $82 \frac{1}{7}$ & 88 & $81 \frac{7}{8}$ & $82 \frac{1}{3}$ & $82 \frac{1}{2}$ & \\
\hline
\end{tabular}

Suppose that $G \in \mathbb{U}_{1}(m)$. Recall that $G$ is a graph of maximum degree three obtainable by attaching some pendent vertices to a cycle $C_{k}$, where $m \leq k \leq 2 m-1$. If $k=m, m+1, m+2$, then there are, respectively, $m, m-1, m-2$ pendent vertices in $G$ outside the cycle $C_{k}$, and thus by Lemma 2.1 (i), we have

$$
\begin{aligned}
K f(G) & \geq \begin{cases}\frac{1}{3}\left(m^{3}+6 m^{2}-4 m\right) & \text { if } k=m \\
\frac{1}{3}\left(m^{3}+7 m^{2}-11 m+6-\frac{3}{m+1}\right) & \text { if } k=m+1 \\
\frac{1}{3}\left(m^{3}+8 m^{2}-20 m+30-\frac{60}{m+2}\right) & \text { if } k=m+2\end{cases} \\
& >6 m^{2}-13 m+4=K f\left(U_{2 m, m}\right) .
\end{aligned}
$$

If $m+3 \leq k \leq 2 m-1$, then by Lemma 2.8, $K f(G) \geq \frac{1}{12} h(k)$, where $h(k)=-k^{3}+$ $4 m k^{2}-(24 m-13) k+48 m^{2}-28 m$. Clearly, $h(k)=-3 k^{2}+8 m k-24 m+13$. Note that $h^{\prime}(m+3)=5 m^{2}-18 m-14>0$ and $h^{\prime}(2 m-1)=4 m^{2}-20 m+10>0$. This implies that $h^{\prime}(k)>0$ for $m+3 \leq k \leq 2 m-1$, i.e., $h(k)$ is increasing for $k$ with $m+3 \leq k \leq 2 m-1$. 
Table 4: The Kirchhoff indices of the graphs $U(k, t, 0, j)$ in $\mathbb{U}(12,6)$.

\begin{tabular}{|c||c|c|c|c|c|c|}
\hline Graphs & $(3,1 ; 4)$ & $(3,3 ; 3)$ & $(4,0 ; 4)$ & $(4,2 ; 3)$ & $(4,4 ; 2)$ & $(5,1 ; 3)$ \\
\hline Kirchhoff indices & 149 & 150 & 149 & $145 \frac{1}{4}$ & 146 & 142 \\
\hline Graphs & $(5,3 ; 2)$ & $(5,5 ; 1)$ & $(6,0 ; 3)$ & $(6,2 ; 2)$ & $(6,4 ; 1)$ & $(6,6 ; 0)$ \\
\hline Kirchhoff indices & $142 \frac{1}{5}$ & 142 & $145 \frac{1}{2}$ & $140 \frac{2}{3}$ & $140 \frac{1}{6}$ & 136 \\
\hline Graphs & $(7,1 ; 2)$ & $(7,3 ; 1)$ & $(7,5 ; 0)$ & $(8,0 ; 2)$ & $(8,2 ; 1)$ & $(8,4 ; 0)$ \\
\hline Kirchhoff indices & 141 & $138 \frac{4}{7}$ & $135 \frac{6}{7}$ & 146 & $139 \frac{5}{8}$ & $135 \frac{1}{2}$ \\
\hline Graphs & $(9,1 ; 1)$ & $(9,3 ; 0)$ & $(10,0 ; 1)$ & $(10,2 ; 0)$ & $(11,1 ; 0)$ & $(12,0 ; 0)$ \\
\hline Kirchhoff indices & 142 & $136 \frac{1}{3}$ & $146 \frac{1}{2}$ & $138 \frac{2}{5}$ & 141 & 143 \\
\hline
\end{tabular}

Table 5: The Kirchhoff indices of the graphs $U(k, t, 0, j)$ in $\mathbb{U}(14,7)$.

\begin{tabular}{|c||c|c|c|c|c|c|}
\hline Graphs & $(3,1 ; 5)$ & $(3,3 ; 4)$ & $(4,0 ; 5)$ & $(4,2 ; 4)$ & $(4,4 ; 3)$ & $(5,1 ; 4)$ \\
\hline Kirchhoff indices & $214 \frac{2}{3}$ & $216 \frac{1}{3}$ & 215 & $210 \frac{3}{4}$ & 213 & 207 \\
\hline Graphs & $(5,3 ; 3)$ & $(5,5 ; 2)$ & $(6,0 ; 4)$ & $(6,2 ; 3)$ & $(6,4 ; 2)$ & $(6,6 ; 1)$ \\
\hline Kirchhoff indices & $208 \frac{2}{5}$ & 211 & $212 \frac{1}{6}$ & 207 & $208 \frac{5}{6}$ & $208 \frac{1}{3}$ \\
\hline Graphs & $(7,1 ; 3)$ & $(7,3 ; 2)$ & $(7,5 ; 1)$ & $(7,7 ; 0)$ & $(8,0 ; 3)$ & $(8,2 ; 2)$ \\
\hline Kirchhoff indices & 208 & 207 & 208 & 203 & 216 & $209 \frac{3}{8}$ \\
\hline Graphs & $(8,4 ; 1)$ & $(8,6 ; 0)$ & $(9,1 ; 2)$ & $(9,3 ; 1)$ & $(9,5 ; 0)$ & $(10,0 ; 2)$ \\
\hline Kirchhoff indices & 208 & $204 \frac{7}{8}$ & $213 \frac{2}{3}$ & $209 \frac{5}{9}$ & $206 \frac{1}{9}$ & $222 \frac{1}{2}$ \\
\hline Graphs & $(10,2 ; 1)$ & $(10,4 ; 0)$ & $(11,1 ; 1)$ & $(11,3 ; 0)$ & $(12,0 ; 1)$ & $(12,2 ; 0)$ \\
\hline Kirchhoff indices & $214 \frac{1}{5}$ & $208 \frac{1}{2}$ & 220 & $212 \frac{9}{20}$ & $227 \frac{2}{3}$ & $217 \frac{7}{12}$ \\
\hline Graphs & $(13,1 ; 0)$ & $(14,0 ; 0)$ & & & & \\
\hline Kirchhoff indices & 223 & $227 \frac{1}{2}$ & & & & \\
\hline
\end{tabular}

Thus

$$
K f(G) \geq \frac{1}{12} h(m+3)=\frac{1}{4}\left(m^{3}+13 m^{2}-26 m+4\right)>6 m^{2}-13 m+4=K f\left(U_{2 m, m}\right) .
$$

Now suppose that $G \in \mathbb{U}_{2}(m)$. Denote by $x$ a pendent vertex in $G$ whose unique neighbor $y$ is of degree two, and $z$ the neighbor of $y$ different from $x$ in $G$. Obviously, $x y \in M$. Then $G-x-y \in \mathbb{U}(2 m-2, m-1)$, and thus by Lemma 2.7 and the induction 
Table 6: The Kirchhoff indices of the graphs $U(k, t, 0, j)$ in $\mathbb{U}(16,8)$.

\begin{tabular}{|c||c|c|c|c|c|c|}
\hline Graphs & $(3,1 ; 6)$ & $(3,3 ; 5)$ & $(4,0 ; 6)$ & $(4,2 ; 5)$ & $(4,4 ; 4)$ & $(5,1 ; 5)$ \\
\hline Kirchhoff indices & $292 \frac{1}{3}$ & $293 \frac{2}{3}$ & 293 & $288 \frac{1}{4}$ & 292 & 284 \\
\hline Graphs & $(5,3 ; 4)$ & $(5,5 ; 3)$ & $(6,0 ; 5)$ & $(6,2 ; 4)$ & $(6,4 ; 3)$ & $(6,6 ; 2)$ \\
\hline Kirchhoff indices & $286 \frac{3}{5}$ & 292 & $290 \frac{5}{6}$ & $285 \frac{1}{3}$ & $289 \frac{1}{2}$ & $292 \frac{2}{3}$ \\
\hline Graphs & $(7,1 ; 4)$ & $(7,3 ; 3)$ & $(7,5 ; 2)$ & $(7,7 ; 1)$ & $(8,0 ; 4)$ & $(8,2 ; 3)$ \\
\hline Kirchhoff indices & 287 & $287 \frac{3}{7}$ & $292 \frac{1}{7}$ & 292 & 298 & $291 \frac{1}{8}$ \\
\hline Graphs & $(8,4 ; 2)$ & $(8,6 ; 1)$ & $(8,8 ; 0)$ & $(9,1 ; 3)$ & $(9,3 ; 2)$ & $(9,5 ; 1)$ \\
\hline Kirchhoff indices & $292 \frac{1}{2}$ & $294 \frac{1}{8}$ & 288 & $297 \frac{1}{3}$ & $294 \frac{7}{9}$ & $295 \frac{5}{9}$ \\
\hline Graphs & $(9,7 ; 0)$ & $(10,0 ; 3)$ & $(10,2 ; 2)$ & $(10,4 ; 1)$ & $(10,6 ; 0)$ & $(11,1 ; 2)$ \\
\hline Kirchhoff indices & $292 \frac{5}{9}$ & $310 \frac{1}{2}$ & 302 & $299 \frac{3}{10}$ & 296 & 311 \\
\hline Graphs & $(11,3 ; 1)$ & $(11,5 ; 0)$ & $(12,0 ; 2)$ & $(12,2 ; 1)$ & $(12,4 ; 0)$ & $(13,1 ; 1)$ \\
\hline Kirchhoff indices & $305 \frac{1}{11}$ & $300 \frac{5}{11}$ & $324 \frac{1}{3}$ & $314 \frac{1}{12}$ & $306 \frac{2}{3}$ & 324 \\
\hline Graphs & $(13,3 ; 0)$ & $(14,0 ; 1)$ & $(14,2 ; 0)$ & $(15,1 ; 0)$ & $(16,0 ; 0)$ & \\
\hline Kirchhoff indices & $314 \frac{7}{13}$ & $335 \frac{1}{2}$ & $323 \frac{3}{7}$ & $332 \frac{1}{3}$ & 340 & \\
\hline
\end{tabular}

hypothesis, we have

$$
\begin{aligned}
K f(G) & \geq K f(G-x-y)+12 m-19 \\
& \geq K f\left(U_{2 m-2, m-1}\right)+12 m-19 \\
& =6 m^{2}-13 m+4=K f\left(U_{2 m, m}\right)
\end{aligned}
$$

with equalities if and only if $G \cong U_{2 m, m}$.

Then the result for $m \geq 9$ follows easily.

The remainder of the paper will focus on the minimum Kirchhoff index among the graphs in $\mathbb{U}(n, m)$, where $n>2 m$ and $m \geq 3$.

Lemma 3.1. [2] Let $G \in \mathbb{U}(n, m) \backslash\left\{C_{n}\right\}$, where $n>2 m, m \geq 3$. Then there is a maximum matching $M$ and a pendent vertex $u$ of $G$ such that $u$ is not $M$-saturated.

For a given graph $G \in \mathbb{U}(n, m) \backslash\left\{C_{n}\right\}$, where $n>2 m, m \geq 3$, by Lemma 3.1, there is a maximum matching $M$ and a pendent vertex which is not $M$-saturated, after deleting this pendent vertex, we can get a graph in $\mathbb{U}(n-1, m)$. Repeating the process until it is exhausted, the resulting graph is denoted by $G_{0}$. Note that $G_{0} \in \mathbb{U}(2 m, m)$. Let $n_{0}=\left|G_{0}\right|$. Furthermore, for the vertex $u \in V\left(G_{0}\right)$ satisfying $K f_{G_{0}}(u)$ is minimum, let $G_{0}^{*}$ be the graph obtained from $G_{0}$ by attaching $n-n_{0}$ pendent vertices to $u$. 
Lemma 3.2. Let $G \in \mathbb{U}(n, m) \backslash\left\{C_{n}\right\}$, where $n>2 m, m \geq 3$.

(i) Then $K f(G) \geq K f\left(G_{0}^{*}\right)$. In particular, if $u$ is the unique vertex in $G_{0}$ such that $K f_{G_{0}}(u)$ is minimum, then $K f(G) \geq K f\left(G_{0}^{*}\right)$ with equality if and only if $G \cong G_{0}^{*}$.

(ii) If $G_{0} ¥ U_{n_{0}, m}$ and $K f\left(G_{0}\right) \geq K f\left(U_{n_{0}, m}\right)$, then $K f(G)>K f\left(U_{n, m}\right)$.

Proof. Similar to the proof of Lemma 2.4, and by Lemma 2.3 repeatedly, (i) follows easily. On the other hand, by Lemmas 2.3 and 2.6, $K f\left(G_{0}^{*}\right)>K f\left(U_{n, m}\right)$ follows from the hypothesis that $G_{0} \nsucceq U_{n_{0}, m}$ and $K f\left(G_{0}\right) \geq K f\left(U_{n_{0}, m}\right)$. Now together with $K f(G) \geq$ $K f\left(G_{0}^{*}\right)$, we can get $K f(G)>K f\left(U_{n, m}\right)$.

The following lemma reveal the possible graph with the minimum Kirchhoff index among the graphs in $\mathbb{U}(n, m) \backslash\left\{C_{n}\right\}$, where $n>2 m$ and $3 \leq m \leq 7$.

Lemma 3.3. If $G$ is a graph in $\mathbb{U}(n, m) \backslash\left\{C_{n}\right\}$ with the minimum Kirchhoff index, where $n>2 m$ and $3 \leq m \leq 7$, then $G_{0} \cong U(k, t, 0, j)$ with $k+t+2 j=n_{0}, k \geq 3, k \geq t \geq 0$ and $j \geq 0$.

Proof. Let $G \in \mathbb{U}(n, m) \backslash\left\{C_{n}\right\}$. Denote by $k$ the length of the unique cycle of $G$. Suppose that there are $t$ pendent vertices of $G$ whose unique neighbors are all on the unique cycle of $G$. Note that $G_{0} \in \mathbb{U}(2 m, m)$, i.e., $G_{0} \in \mathbb{U}_{1}(m) \cup \mathbb{U}_{2}(m)$.

Case 1. $G_{0} \in \mathbb{U}_{1}(m)$.

First, by Lemma 3.2 (i), we have $K f(G) \geq K f\left(G_{0}^{*}\right)$. Next, by Lemma 2.2, we have $K f\left(G_{0}\right) \geq K f(U(k, t))$ with equality if and only if $\left.G_{0} \cong U(k, t)\right)$, and thus by Lemma 2.1 (ii) and Lemma 2.3 , we have $K f\left(G_{0}^{*}\right) \geq K f(U(k, t, i, 0))$ with equality if and only if $G_{0}^{*} \cong$ $U(k, t, i, 0))$, where $k+t+i=n$ and $i \geq 1$. Now it follows that $K f(G) \geq K f(U(k, t, i, 0))$ with equality if and only if $G \cong U(k, t, i, 0)$.

Case 2. $G_{0} \in \mathbb{U}_{2}(m)$.

Recall that, starting from $G_{0}$, deleting the pendent $P_{2}$ 's repeatedly, until there is no pendent $P_{2}$, the resulting graph is denoted by $\bar{G}_{0}$. Let $\overline{n_{0}}=\left|\bar{G}_{0}\right|$. Clearly, $\bar{G}_{0} \in$ $\mathbb{U}_{1}\left(\frac{\overline{n_{0}}}{2}\right) \cup\left\{C_{\overline{n_{0}}}\right\}$.

Suppose that $u$ is a vertex in $\bar{G}_{0}$ satisfying $K f_{\bar{G}_{0}}(u)$ is minimum, and let $H$ be the graph obtained from $\bar{G}_{0}$ by attaching $i$ pendent vertices and $j$ paths on two vertices to $u$.

Similar to the proof of Lemma 2.4, and by Lemma 2.3 repeatedly, $K f(G) \geq K f(H)$ follows easily.

On the other hand, recall that $\bar{G}_{0} \in \mathbb{U}_{1}\left(\frac{\overline{n_{0}}}{2}\right) \cup\left\{C_{\overline{n_{0}}}\right\}$, by Lemma 2.2 , we have $K f\left(\bar{G}_{0}\right) \geq$ $K f(U(k, t))$ with equality if and only if $\left.\bar{G}_{0}^{2} \cong U(k, t)\right)$, where $k+t=\overline{n_{0}}$, and thus by Lemma 2.1 (ii) and Lemma 2.3, we have $K f(H) \geq K f(U(k, t, i, j))$ with equality if and only if $H \cong U(k, t, i, j)$, where $k+t+i+2 j=n$.

Now it follows that $K f(G) \geq K f(U(k, t, i, j))$ with equality if and only if $G \cong$ $U(k, t, i, j)$.

Combining Cases 1 and 2, we have $K f(G) \geq K f(U(k, t, i, j))$ with equality if and only if $G \cong U(k, t, i, j)$, and $G \cong U(k, t, i, j)$ implies that $G_{0} \cong U(k, t, 0, j)$. 
Now we determine the minimum Kirchhoff index among the unicyclic graphs with given matching number.

Theorem 3.2. Among the graphs in $\mathbb{U}(n, m)$ with $2 \leq m \leq\left\lfloor\frac{n}{2}\right\rfloor$,

(i) for $m=2, C_{n}$ for $n=4,5, U(4,1, n-5,0)$ for $6 \leq n \leq 11, U(3,1,8,0)$ and $U(4,1,7,0)$ for $n=12$, and $U(3,1, n-4,0)$ for $n \geq 13$ are the unique graphs with the minimum Kirchhoff indices, which are equal to $\frac{n^{3}-n}{12}$ for $n=4,5, \frac{1}{2}\left(2 n^{2}-5 n-2\right)$ for $6 \leq n \leq 11,113$ for $n=12$, and $\frac{1}{3}\left(3 n^{2}-8 n+3\right)$ for $n \geq 13$;

(ii) for $m=3, C_{n}$ for $n=6,7$ and $U_{n, 3}$ for $n \geq 8$ are the unique graphs with the minimum Kirchhoff indices, which are equal to $\frac{n^{3}-n}{12}$ for $n=6,7$ and $n^{2}-2 n-5$ for $n \geq 8$

(iii) for $m=4, C_{8}$ for $n=8, U(7,1,1,0)$ and $C_{9}$ for $n=9, U(7,1,2,0)$ for $n=10$, $U(6,2,3,0)$ and $U(7,1,3,0)$ for $n=11, U(6,2, n-8,0)$ for $n=12,13, U_{14,4}$ and $U(6,2,6,0)$ for $n=14$, and $U_{n, m}$ for $n \geq 15$ are the unique graphs with the minimum Kirchhoff indices, which are equal to 42 for $n=8,60$ for $n=9$, 79 for $n=10$, 100 for $n=11, \frac{1}{3}\left(3 n^{2}-n-52\right)$ for $n=12,13,174$ for $n=14$, and $n^{2}-n-8$ for $n \geq 15$;

(iv) for $m=5, U(8,2)$ for $n=10, U(7,3, n-10,0)$ for $11 \leq n \leq 13, U_{14,5}, U(6,2,4,1)$ and $U(7,3,4,0)$ for $n=14$, and $U_{n, 5}$ for $n \geq 15$ are the unique graphs with the minimum Kirchhoff indices, which are equal to $81 \frac{7}{8}$ for $n=10, \frac{1}{7}\left(7 n^{2}+12 n-245\right)$ for $11 \leq n \leq 13,185$ for $n=14$, and $n^{2}-11$ for $n \geq 15$;

(v) for $m=6, U(8,4, n-12,0)$ for $n=12,13, U_{14,6}, U(6,2,2,2)$ and $U(7,3,2,1)$ for $n=14$, and $U_{n, 6}$ for $n \geq 15$ are the unique graphs with the minimum Kirchhoff indices, which are equal to $\frac{1}{4}\left(4 n^{2}+19 n-262\right)$ for $n=12,13,196$ for $n=14$, and $n^{2}+n-14$ for $n \geq 15$;

(vi) for $m=7, U(7,7)$ for $n=14$ and $U_{n, 7}$ for $n \geq 15$ are the unique graphs with the minimum Kirchhoff indices, which are equal to 203 for $n=14$ and $n^{2}+2 n-17$ for $n \geq 15$

(vii) for $m \geq 8, U_{n, m}$ for $n \geq 16$ is the unique graph with the minimum Kirchhoff index, which is equal to $n^{2}+n m-5 n-3 m+4$.

Proof. The result for $n=2 m$ follows from Theorem 3.1. Suppose that $n>2 m$. Let $G \in \mathbb{U}(n, m)$.

Case 1. $m=2$.

Clearly, the girth of $G$ is $3,4,5$ for $n=5$, and 3,4 for $n \geq 6$. Then by Lemma 2.8, we have

$$
K f(G) \geq \min \left\{K f(U(3,1,1,0)), K f(U(4,1)), K f\left(C_{5}\right)\right\}
$$




$$
=\min \left\{12 \frac{2}{3}, 11 \frac{1}{2}, 10\right\}=10
$$

for $n=5$, and

$$
\begin{aligned}
K f(G) & \geq \min \{K f(U(3,1, n-4,0)), K f(U(4,1, n-5,0))\} \\
& =\min \left\{\frac{1}{3}\left(3 n^{2}-8 n+3\right), \frac{1}{2}\left(2 n^{2}-5 n-2\right)\right\}
\end{aligned}
$$

for $n \geq 6$. Thus $C_{5}$ for $n=5, U(4,1, n-5,0)$ for $6 \leq n \leq 11, U(3,1,8,0)$ and $U(4,1,7,0)$ for $n=12$, and $U(3,1, n-4,0)$ for $n \geq 13$ are the unique graphs in $\mathbb{U}(n, 2)$ with the minimum Kirchhoff indices.

Case 2. $m=3$.

If $G \cong C_{n}$, then $n=7$, and by Eq. (3), we have $K f(G)=28$.

Suppose that $G \approx C_{n}$. If $G_{0} \cong U_{6,3}$, then by Lemma 3.2 (i), we have $K f(G) \geq$ $K f\left(U_{n, 3}\right)$ with equality if and only if $G \cong U_{n, 3}$. Suppose that $G_{0} \neq U_{6,3}$. If $K f\left(G_{0}\right) \geq$ $K f\left(U_{6,3}\right)$, then by Lemma 3.2 (ii), we have $K f(G)>K f\left(U_{n, 3}\right)$. If $K f\left(G_{0}\right)<K f\left(U_{6,3}\right)$, then by Lemma 3.3 and Table 1, we assume that $G_{0}=C_{6}$, and thus by Lemma 3.2 (i), we have $K f(G) \geq K f(U(6,1, n-7,0))$ with equality if and only if $G \cong U(6,1, n-7,0)$.

Therefore for $n=7$,

$$
K f(G) \geq \min \left\{K f\left(U_{7,3}\right), K f(U(6,1)), K f\left(C_{7}\right)\right\}=\min \left\{30,29 \frac{1}{3}, 28\right\}=28
$$

with equality if and only if $G \cong C_{7}$, and for $n \geq 8$,

$$
\begin{aligned}
K f(G) & \geq \min \left\{K f\left(U_{n, 3}\right), K f(U(6,1, n-7,0))\right\} \\
& =\min \left\{n^{2}-2 n-5, n^{2}-\frac{7}{6} n-\frac{23}{2}\right\}=n^{2}-2 n-5
\end{aligned}
$$

with equality if and only if $G \cong U_{n, 3}$.

Case 3. $m=4$.

If $G \cong C_{n}$, then $n=9$, and by Eq. (3), we have $K f(G)=60$.

Suppose that $G ¥ C_{n}$. If $G_{0} \cong U_{8,4}$, then by Lemma 3.2 (i), we have $K f(G) \geq$ $K f\left(U_{n, 4}\right)$ with equality if and only if $G \cong U_{n, 4}$. Suppose that $G_{0} \neq U_{8,4}$. If $K f\left(G_{0}\right) \geq$ $K f\left(U_{8,4}\right)$, then by Lemma 3.2 (ii), we have $K f(G)>K f\left(U_{n, 4}\right)$. If $K f\left(G_{0}\right)<K f\left(U_{8,4}\right)$, then by Lemma 3.3 and Table 2 , we assume that $G_{0}=U(5,3,0,0), U(6,2,0,0), U(7,1,0,0)$ or $U(8,0,0,0)$, and thus by Lemma 3.2 (i), we have

$$
\begin{aligned}
K f(G) \geq & \min \{K f(U(5,3, n-8,0)), K f(U(6,2, n-8,0)), \\
& K f(U(7,1, n-8,0)), K f(U(8,0, n-8,0))\} .
\end{aligned}
$$

Now the result for $m=4$ follows from Table 7 easily.

Case 4. $m=5$.

If $G \cong C_{n}$, then $n=11$, and by Eq. (3), we have $K f(G)=110$. 
Table 7: The graphs in $\mathbb{U}(n, 4)$ and their Kirchhoff indices.

\begin{tabular}{|c|cccccccc|}
\hline \multirow{2}{*}{ Graphs } & \multicolumn{7}{|c|}{ Kirchhoff indices } \\
\cline { 2 - 9 } & $n$ & 9 & 10 & 11 & 12 & 13 & 14 & 15 \\
\hline$U_{n, 4}$ & $n^{2}-n-8$ & 64 & 82 & 102 & 124 & 148 & $\mathbf{1 7 4}$ & $\mathbf{2 0 2}$ \\
\hline$U(5,3, n-8,0)$ & $n^{2}-\frac{2}{5} n-15$ & $62 \frac{2}{5}$ & 81 & $101 \frac{3}{5}$ & $124 \frac{1}{5}$ & $148 \frac{4}{5}$ & $175 \frac{2}{5}$ & 204 \\
\hline$U(6,2, n-8,0)$ & $n^{2}-\frac{1}{3} n-\frac{52}{3}$ & $60 \frac{2}{3}$ & $79 \frac{1}{3}$ & $\mathbf{1 0 0}$ & $\mathbf{1 2 2} \frac{\mathbf{2}}{\mathbf{3}}$ & $\mathbf{1 4 7} \frac{1}{\mathbf{3}}$ & $\mathbf{1 7 4}$ & $202 \frac{2}{3}$ \\
\hline$U(7,1, n-8,0)$ & $n^{2}-21$ & $\mathbf{6 0}$ & $\mathbf{7 9}$ & $\mathbf{1 0 0}$ & 123 & 148 & 175 & 204 \\
\hline$U(8,0, n-8,0)$ & $n^{2}+\frac{3}{2} n-34$ & $60 \frac{1}{2}$ & 81 & $103 \frac{1}{2}$ & 128 & $154 \frac{1}{2}$ & 183 & $213 \frac{1}{2}$ \\
\hline$C_{9}$ & $\mathbf{6 0}$ & & & & & \\
\hline
\end{tabular}

Suppose that $G \neq C_{n}$. If $G_{0} \cong U_{10,5}$, then by Lemma 3.2 (i), we have $K f(G) \geq$ $K f\left(U_{n, 5}\right)$ with equality if and only if $G \cong U_{n, 5}$. Suppose that $G_{0} \neq U_{10,5}$. If $K f\left(G_{0}\right) \geq$ $K f\left(U_{10,5}\right)$, then by Lemma 3.2 (ii), we have $K f(G)>K f\left(U_{n, 5}\right)$. If $K f\left(G_{0}\right)<K f\left(U_{10,5}\right)$, then by Lemma 3.3 and Table 3 , we assume that $G_{0}=U(5,3,0,1), U(5,5,0,0), U(6,2,0,1)$, $U(6,4,0,0), U(7,1,0,1), U(7,3,0,0), U(8,0,0,1), U(8,2,0,0), U(9,1,0,0)$ or $U(10,0,0,0)$, and thus by Lemma 3.2 (i), we have

$$
\begin{aligned}
K f(G) \geq & \min \{K f(U(5,3, n-10,1)), K f(U(5,5, n-10,0)), K f(U(6,2, n-10,1)), \\
& K f(U(6,4, n-10,0)), K f(U(7,1, n-10,1)), K f(U(7,3, n-10,0)), \\
& K f(U(8,0, n-10,1)), K f(U(8,2, n-10,0)), K f(U(9,1, n-10,0)), \\
& K f(U(10,0, n-10,0))\} .
\end{aligned}
$$

Now the result for $m=5$ follows from Table 8 easily.

Case 5. $m=6$.

If $G \cong C_{n}$, then $n=13$, and by Eq. (3), we have $K f(G)=182$.

Suppose that $G \neq C_{n}$. If $G_{0} \cong U_{12,6}$, then by Lemma 3.2 (i), we have $K f(G) \geq$ $K f\left(U_{n, 6}\right)$ with equality if and only if $G \cong U_{n, 6}$. Suppose that $G_{0} \neq U_{12,6}$. If $K f\left(G_{0}\right) \geq$ $K f\left(U_{12,6}\right)$, then by Lemma 3.2 (ii), we have $K f(G)>K f\left(U_{n, 6}\right)$. If $K f\left(G_{0}\right)<K f\left(U_{12,6}\right)$, then by Lemma 3.3 and Table 4 , we assume that $G_{0}=U(6,2,0,2), U(6,4,0,1), U(6,6,0,0)$, $U(7,1,0,2), U(7,3,0,1), U(7,5,0,0), U(8,2,0,1), U(8,4,0,0), U(9,3,0,0), U(10,2,0,0)$ or $U(11,1,0,0)$, and thus by Lemma 3.2 (i), we have

$$
\begin{aligned}
K f(G) \geq & \min \{K f(U(6,2, n-12,2)), K f(U(6,4, n-12,1)), K f(U(6,6, n-12,0)), \\
& K f(U(7,1, n-12,2)), K f(U(7,3, n-12,1)), K f(U(7,5, n-12,0)), \\
& K f(U(8,2, n-12,1)), K f(U(8,4, n-12,0)), K f(U(9,3, n-12,0)), \\
& K f(U(10,2, n-12,0)), K f(U(11,1, n-12,0))\} .
\end{aligned}
$$

Now the result for $m=6$ follows from Table 9 easily. 
Table 8: The graphs in $\mathbb{U}(n, 5)$ and their Kirchhoff indices.

\begin{tabular}{|c|cccccc|}
\hline \multirow{2}{*}{ Graphs } & \multicolumn{7}{|c|}{ Kirchhoff indices } \\
\cline { 2 - 7 } & $n$ & 11 & 12 & 13 & 14 & 15 \\
\hline$U_{n, 5}$ & $n^{2}-11$ & 110 & 133 & 158 & $\mathbf{1 8 5}$ & $\mathbf{2 1 4}$ \\
\hline$U(5,3, n-10,1)$ & $n^{2}+\frac{3}{5} n-18$ & $109 \frac{3}{5}$ & $133 \frac{1}{5}$ & $158 \frac{4}{5}$ & $186 \frac{2}{5}$ & 216 \\
\hline$U(5,5, n-10,0)$ & $n^{2}+2 n-35$ & 108 & 133 & 160 & 189 & 220 \\
\hline$U(6,2, n-10,1)$ & $n^{2}+\frac{2}{3} n-\frac{61}{3}$ & 108 & $131 \frac{2}{3}$ & $157 \frac{1}{3}$ & $\mathbf{1 8 5}$ & $214 \frac{2}{3}$ \\
\hline$U(6,4, n-10,0)$ & $n^{2}+\frac{11}{6} n-\frac{209}{6}$ & $106 \frac{1}{3}$ & $131 \frac{1}{6}$ & 158 & $186 \frac{5}{6}$ & $217 \frac{2}{3}$ \\
\hline$U(7,1, n-10,1)$ & $n^{2}+n-24$ & 108 & 132 & 158 & 186 & 216 \\
\hline$U(7,3, n-10,0)$ & $n^{2}+\frac{12}{7} n-\frac{245}{7}$ & $\mathbf{1 0 4} \frac{\mathbf{6}}{7}$ & $\mathbf{1 2 9} \frac{4}{7}$ & $\mathbf{1 5 6} \frac{\mathbf{2}}{7}$ & $\mathbf{1 8 5}$ & $215 \frac{5}{7}$ \\
\hline$U(8,0, n-10,1)$ & $n^{2}+\frac{5}{2} n-37$ & $111 \frac{1}{2}$ & 137 & $164 \frac{1}{2}$ & 194 & $225 \frac{1}{2}$ \\
\hline$U(8,2, n-10,0)$ & $n^{2}+\frac{19}{8} n-\frac{335}{8}$ & $105 \frac{1}{4}$ & $130 \frac{5}{8}$ & 158 & $187 \frac{3}{8}$ & $218 \frac{3}{4}$ \\
\hline$U(9,1, n-10,0)$ & $n^{2}+\frac{10}{3} n-51$ & $106 \frac{2}{3}$ & 133 & $161 \frac{1}{3}$ & $191 \frac{2}{3}$ & 224 \\
\hline$U(10,0, n-10,0)$ & $n^{2}+\frac{11}{2} n-\frac{145}{2}$ & 109 & $137 \frac{1}{2}$ & 168 & $200 \frac{1}{2}$ & 235 \\
\hline$C_{11}$ & & 110 & & & & \\
\hline
\end{tabular}

Case 6. $m=7$.

If $G \cong C_{n}$, then $n=15$, and by Eq. (3), we have $K f(G)=280$.

Suppose that $G \neq C_{n}$. If $G_{0} \cong U_{14,7}$, then by Lemma 3.2 (i), we have $K f(G) \geq$ $K f\left(U_{n, 7}\right)$ with equality if and only if $G \cong U_{n, 7}$. Suppose that $G_{0} \nsucceq U_{14,7}$. If $K f\left(G_{0}\right) \geq$ $K f\left(U_{14,7}\right)$, then by Lemma 3.2 (ii), we have $K f(G)>K f\left(U_{n, 7}\right)$. If $K f\left(G_{0}\right)<K f\left(U_{14,7}\right)$, then by Lemma 3.3 and Table 5 , we assume that $G_{0}=U(7,7,0,0), U(8,6,0,0)$ or $U(9,5,0,0)$, and thus by Lemma 3.2 (i), we have

$$
K f(G) \geq \min \{K f(U(7,7, n-14,0)), K f(U(8,6, n-14,0)), K f(U(9,5, n-14,0))\} .
$$

Now the result for $m=7$ follows from Table 10 easily.

Case 7. $m \geq 8$.

If $G \cong C_{n}$, then $n=2 m+1$, and by Eqs. (3) and (5), we have

$$
K f\left(C_{2 m+1}\right)=\frac{1}{3}\left(2 m^{3}+3 m^{2}+m\right)>6 m^{2}-8 m=K f\left(U_{2 m+1, m}\right) .
$$

Suppose that $G ¥ C_{n}$. By Theorem 3.1, $K f\left(G_{0}\right) \geq K f\left(U_{n_{0}, m}\right)$. Furthermore, if $G_{0} \cong$ $U_{n_{0}, m}$, then by Lemma 3.2 (i), we have $K f(G) \geq K f\left(U_{n, m}\right)$ with equality if and only if $G \cong U_{n, m}$, and if $G_{0} \nsucceq U_{n_{0}, m}$, then by Lemma 3.2 (ii), we have $K f(G)>K f\left(U_{n, m}\right)$. Then the result for $m \geq 8$ follows easily. 
Table 9: The graphs in $\mathbb{U}(n, 6)$ and their Kirchhoff indices.

\begin{tabular}{|c|cccc|}
\hline \multirow{2}{*}{ Graphs } & \multicolumn{5}{|c|}{ Kirchhoff indices } \\
\cline { 2 - 5 } & $n$ & 13 & 14 & 15 \\
\hline$U_{n, 6}$ & $n^{2}+n-14$ & 168 & $\mathbf{1 9 6}$ & $\mathbf{2 2 6}$ \\
\hline$U(6,2, n-12,2)$ & $n^{2}+\frac{5}{3} n-\frac{70}{3}$ & $167 \frac{1}{3}$ & $\mathbf{1 9 6}$ & $226 \frac{2}{3}$ \\
\hline$U(6,4, n-12,1)$ & $n^{2}+\frac{17}{6} n-\frac{227}{6}$ & 168 & $197 \frac{5}{6}$ & $229 \frac{2}{3}$ \\
\hline$U(6,6, n-12,0)$ & $n^{2}+\frac{14}{3} n-64$ & $165 \frac{2}{3}$ & $197 \frac{1}{3}$ & 231 \\
\hline$U(7,1, n-12,2)$ & $n^{2}+2 n-27$ & 168 & 197 & 228 \\
\hline$U(7,3, n-12,1)$ & $n^{2}+\frac{19}{7} n-38$ & $166 \frac{2}{7}$ & $\mathbf{1 9 6}$ & $227 \frac{5}{7}$ \\
\hline$U(7,5, n-12,0)$ & $n^{2}+\frac{32}{7} n-63$ & $165 \frac{3}{7}$ & 197 & $230 \frac{4}{7}$ \\
\hline$U(8,2, n-12,1)$ & $n^{2}+\frac{27}{8} n-\frac{359}{8}$ & 168 & $198 \frac{3}{8}$ & $230 \frac{3}{4}$ \\
\hline$U(8,4, n-12,0)$ & $n^{2}+\frac{19}{4} n-\frac{131}{2}$ & $\mathbf{1 6 5} \frac{1}{4}$ & 197 & $230 \frac{3}{4}$ \\
\hline$U(9,3, n-12,0)$ & $n^{2}+\frac{46}{9} n-69$ & $166 \frac{4}{9}$ & $198 \frac{5}{9}$ & $232 \frac{2}{3}$ \\
\hline$U(10,2, n-12,0)$ & $n^{2}+\frac{32}{5} n-\frac{412}{5}$ & $169 \frac{4}{5}$ & $203 \frac{1}{5}$ & $238 \frac{3}{5}$ \\
\hline$U(11,1, n-12,0)$ & $n^{2}+8 n-99$ & 174 & 209 & 246 \\
\hline$C_{13}$ & & 182 & & \\
\hline
\end{tabular}

Acknowledgement. This work was supported by the Research Fund for the Doctoral Program of Higher Education of China (No. 20124407110002), the Science Foundation of Hebei Normal University (no. L2012Q02) and the Guangdong Provincial National Science Foundation of China (no. S2011010005539).

\section{References}

[1] D. Bonchev, A.T. Balaban, X. Liu, D.J. Klein, Molecular cyclicity and centricity of polycyclic graphs, Int. J. Quantum Chem. 50 (1994) 1-20.

[2] A. Chang, F. Tian, On the spectral radius of unicyclic graphs with perfect matching, Linear Algebra Appl. 370 (2003) 237-250.

[3] Z. Du, B. Zhou, Minimum Wiener indices of trees and unicyclic graphs of given matching number, MATCH Commun. Math. Comput. Chem. 63 (2010) 101-112.

[4] A.A. Dobrynin, R. Entringer, I. Gutman, Wiener index of trees: Theory and applications, Acta Appl. Math. 66 (2001) 211-249. 
Table 10: The graphs in $\mathbb{U}(n, 7)$ and their Kirchhoff indices.

\begin{tabular}{|c|cc|}
\hline \multirow{2}{*}{ Graphs } & \multicolumn{2}{|c|}{ Kirchhoff indices } \\
\cline { 2 - 3 } & $n$ & 15 \\
\hline$U_{n, 7}$ & $n^{2}+2 n-17$ & $\mathbf{2 3 8}$ \\
\hline$U(7,7, n-14,0)$ & $n^{2}+8 n-105$ & 240 \\
\hline$U(8,6, n-14,0)$ & $n^{2}+\frac{65}{8} n-\frac{839}{8}$ & 242 \\
\hline$U(9,5, n-14,0)$ & $n^{2}+\frac{74}{9} n-105$ & $243 \frac{1}{3}$ \\
\hline$C_{15}$ & & 280 \\
\hline
\end{tabular}

[5] A.A. Dobrynin, I. Gutman, S. Klavžar, P. Žigert, Wiener index of hexagonal systems, Acta Appl. Math. 72 (2002) 247-294.

[6] A. Ghosh, S. Boyd, A. Saberi, Minimizing effective resitance of graph, SIAM Rev. 50 (2008) 37-66.

[7] D.J. Klein, Resistance-distance sum rules, Croat. Chem. Acta 75 (2002) 633-649.

[8] D.J. Klein, M. Randić, Resistance distance, J. Math. Chem. 12 (1993) 81-95.

[9] I. Lukovits, S. Nikolić, N. Trinajstić, Resistance distance in regular graphs, Int. J. Quantum Chem. 71 (1999) 217-225.

[10] J.L. Palacios, Resistance distance in graphs and random walks, Int. J. Quantum Chem. 81 (2001) 29-33.

[11] Y. Yang, X. Jiang, Unicyclic graphs with extremal Kirchhoff index, MATCH Commun. Math. Comput. Chem. 60 (2008) 107-120.

[12] W. Zhang, H. Deng, The second maximal and minimal Kirchhoff indices of unicyclic graphs, MATCH Commun. Math. Comput. Chem. 61 (2009) 683-695.

[13] B. Zhou, N. Trinajstic, A note on Kirchhoff index, Chem. Phys. Lett. 445 (2008) 120-123.

[14] B. Zhou, N. Trinajstic, On resistance-distance and Kirchhoff index, J. Math. Chem. 46 (2009) 283-289.

[15] B. Zhou, N. Trinajstic, Mathematical properties of molecular descriptors based on distances, Croat. Chem. Acta 83 (2010) 227-242.

[16] B. Zhou, N. Trinajstic, The Kirchhoff index and the matching number, Int. J. Quantum Chem. 109 (2009) 2978-2981. 